\title{
Experiencia preliminar en el tratamiento combinado de metástasis vertebrales mediante radiofrecuencia y cifoplastia en sesión única
}

\author{
G. Buitrago, E. Castro, J. C. Garrido, P. Fernández-García, J. Guzmán de Villoria, F. Fortea y F. Villoria \\ Servicio de Radiodiagnóstico. Sección de Neurorradiología. Hospital General Universitario \\ Gregorio Marañón. Madrid
}

Buitrago G, Castro E, Garrido JC, Fernández-García P, Guzmán de Villoria J, Fortea F, Villoria F. Experiencia preliminar en el tratamiento combinado de metástasis vertebrales mediante radiofrecuencia y cifoplastia en sesión única. Rev Soc Esp Dolor 2013; 20(1): 11-15.

\begin{abstract}
Objectives: Describe our preliminary experience in the treatment of vertebral metastases by radiofrequency and Kyphoplasty combined in one single session.

Material and methods: Four patients with histologically confirmed single spinal metastasis (breast, prostate, lung and myeloma in L1, L5, D12, D12, respectively) were treated. The indication in all cases was pain with a poor response to medical treatment. All patients had pain in the range 6-7 visual analogue scale (VAS). In two cases there was a lytic lesion of the spinal posterior wall. After obtaining informed consent, and under sedation and local anesthetic the procedure took place. The transpedicular approach took place with a $11 \mathrm{G}$ bone puncture system. Two radiofrequency needles were coaxially inserted to carry out an ablation cycle through each pedicle. During the ablation cycle the tip of the ablation neddle stood between the anterior and middle third of the vertebral body, while the second needle was used as thermal sensor with its end to the height of the vertebral posterior wall. The duration of each cycle of ablation was 8 minutes reaching intratumoral temperatures of 70-80 ${ }^{\circ} \mathrm{C}$. Transpedicular Kyphoplasty was performed subsequently.

Results: No complications were reported during or after the procedure and patients were discharged in the first 24 hours. There was an immediate improvement in pain after the procedure (with a VAS 1-2 intensity pain) in all patients. During follow up, analgesic medication was withdrawn in three patients, and
\end{abstract}

there was no evidence of disease progression or recurrence of pain (pain intensity 1 (VAS) in a follow-up in the range of 8-14 months). Clinical and radiological follow-up after discharge could not be performed on a patient.

Conclusion: The use of radio-frequency associated with Kyphoplasty in vertebral metastatic disease can contribute to the management of refractory pain to medical treatment.

Key words: Vertebral metastases. Radiofrequency. Kyphoplasty.

\section{RESUMEN}

Objetivos: Describir nuestra experiencia preliminar en el tratamiento de metástasis vertebrales mediante radiofrecuencia y cifoplastia combinadas en sesión única.

Material y métodos: Se trataron cuatro pacientes con metástasis vertebral única confirmada histológicamente (mama, próstata, pulmón y mieloma en D12, L1, L5 y D12, respectivamente). La indicación en todos los casos fue el dolor con una mala respuesta al tratamiento médico habitual. Todos los pacientes presentaban dolor en el rango 6-7 de la escala visual analógica (EVA). En dos casos existía lesión lítica del muro posterior. Tras la obtención del consentimiento informado se realizó el procedimiento bajo sedación e infiltración anestésica local. Se efectuó abordaje transpedicular bilateral con sistemas de punción ósea 11G. Se insertaron de forma coaxial dos agujas de radiofrecuencia para efectuar un ciclo de ablación por cada pedículo. Durante el ciclo de ablación la punta del dispositivo correspondiente se situó en la unión del tercio medio con el tercio anterior del cuerpo vertebral, empleando la segunda aguja como sensor térmico, con su extremo a la altura del muro posterior. La duración de cada ciclo de ablación fue de 8 minutos, alcanzando temperaturas intratumorales de 70-80 ํ․ A continuación se realizó cifoplastia transpedicular.

Resultados: No se registraron complicaciones intra-periprocedimiento, con alta domiciliaria en las 24 horas siguientes. En todos los pacientes hubo una mejoría inmediata del dolor tras 
el procedimiento (con dolor de intensidad 1-2 de la EVA). En tres pacientes se retiró progresivamente la medicación analgésica, sin evidencia en ninguno de ellos de progresión local de la enfermedad ni recurrencia-aumento del dolor en el seguimiento (dolor de intensidad 1 de la EVA en un seguimiento en el rango de 8-14 meses). En un paciente no se pudo efectuar seguimiento clínico-radiológico posterior al alta.

Conclusión: El empleo de radiofrecuencia asociada a cifoplastia en la enfermedad metastásica vertebral puede contribuir al manejo del dolor refractario al tratamiento médico y al control local de la enfermedad.

Palabras clave: Metástasis vertebrales. Radiofrecuencia. Cifoplastia.

\section{INTRODUCCIÓN}

El dolor secundario a la enfermedad ósea metastásica raquídea representa una causa importante de deterioro de la calidad de vida en pacientes oncológicos, por lo que su adecuado manejo es una prioridad. También resulta fundamental el control de la progresión local de la enfermedad, con el fin de evitar fracturas patológicas y/o posibles cuadros de compresión medular o radicular.

El manejo paliativo de las metástasis vertebrales se basa en una combinación de tratamientos que incluyen las terapias médicas (corticoides, analgesia, quimioterapia, tratamiento hormonal y bifosfonatos), la cirugía y/o la radioterapia $(1,2)$. Estudios recientes sugieren que los tratamientos percutáneos, mínimamente invasivos, pueden contribuir al manejo de estos pacientes $(3,4,6-13)$.

El objetivo de este estudio es describir los aspectos técnicos del procedimiento y nuestra experiencia preliminar en el tratamiento de lesiones vertebrales metastásicas, mediante el empleo combinado de radiofrecuencia (RF) y cementación posterior (cifoplastia), en sesión única.

\section{MATERIAL Y MÉTODOS}

Se trataron cuatro pacientes con lesiones metastásicas en la columna vertebral, dos de ellos con afectación de D12 (mama y mieloma) y los otros dos con afectación de L1 (próstata) y L5 (pulmón). Todas las lesiones habían sido biopsiadas y confirmadas mediante histología. La lesión vertebral constituía la única afectación metastásica conocida en todos los pacientes. Las exploraciones radiológicas previas (RM y TC) habían mostrado afectación metastásica difusa del cuerpo vertebral, sin masa de partes blandas asociada y sin lesión neurológica. Dos de los pacientes presentaban lesión lítica del muro posterior, sospechada en estudio de RM y confirmada mediante TC.

Todos los pacientes habían recibido previamente radioterapia como tratamiento local de sus lesiones ver- tebrales, así como quimioterapia sistémica. Sin embargo, persistía el dolor con mala respuesta al tratamiento analgésico. Los cuatro pacientes presentaban, previo al procedimiento, un dolor en el rango 6-7 de la escala visual analógica (EVA).

La indicación se estableció con intención paliativa, siendo los objetivos planteados la mejoría del dolor y una potencial mejoría en el control local de la enfermedad.

Los procedimientos se realizaron, tras la obtención del consentimiento informado, en una sala de radiología vascular intervencionista dotada de angiógrafo digital. Se llevaron a cabo bajo sedación e infiltración anestésica local, con empleo de profilaxis antibiótica (cefazolina $2 \mathrm{~g}$ intravenosos monodosis) 30 minutos antes del inicio del procedimiento.

El tratamiento se realizó en posición decúbito prono, efectuando punción transpedicular bilateral con agujas de punción ósea de $11 \mathrm{G}$ (Angiotech). Posteriormente se introdujeron de forma coaxial 2 agujas de RF (Valleylab tipo cool tip, Covidien ${ }^{\circledR}$ ) provistas de puntas activas de $1 \mathrm{~cm}$. La aguja empleada para la ablación se avanzó en el cuerpo vertebral, posicionando la punta en la unión del tercio anterior con el medio del cuerpo vertebral, sin necesidad de fresado óseo. El extremo distal de la segunda aguja se situó a la altura del muro posterior, con el objetivo de controlar las potenciales variaciones térmicas durante la RF (Fig. 1). Durante la ablación el objetivo fue alcanzar temperaturas en el rango de $70-80{ }^{\circ} \mathrm{C}$ durante al menos 8 minutos, con empleo de refrigeración simultánea con suero. Posteriormente se repitió la operación con inversión de la posición de las agujas de RF, de forma que se efectuó una segunda ablación por el pedículo contralateral con los mismos parámetros. La aguja empleada como sensor térmico no registró en ningún momento aumentos de temperatura superiores a $1{ }^{\circ} \mathrm{C}$ en el muro posterior.

Una vez finalizado el procedimiento de RF, se intercambiaron las agujas de acceso de $11 \mathrm{G}$ y se realizó cifoplastia,

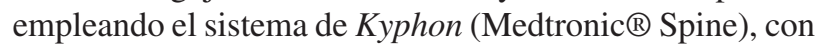
empleo de balones de 1,5 cm en todos los casos (Fig. 2).

\section{RESULTADOS}

No se registraron complicaciones intra o periprocedimiento, con una duración total del procedimiento inferior a 60 minutos en todos los casos. El alta domiciliaria se efectuó al día siguiente. En los cuatro casos se obtuvo, en las 24 horas siguientes al procedimiento, una mejoría del dolor (con una intensidad del dolor tras el procedimiento en el rango de 1-2 de la EVA), lo que permitió la retirada progresiva de la medicación analgésica. En tres pacientes no se detectó reaparición-aumento posterior del dolor en un rango de seguimiento de entre 8 y 14 meses (dolor de intensidad 1 de la EVA). No fue posible realizar 

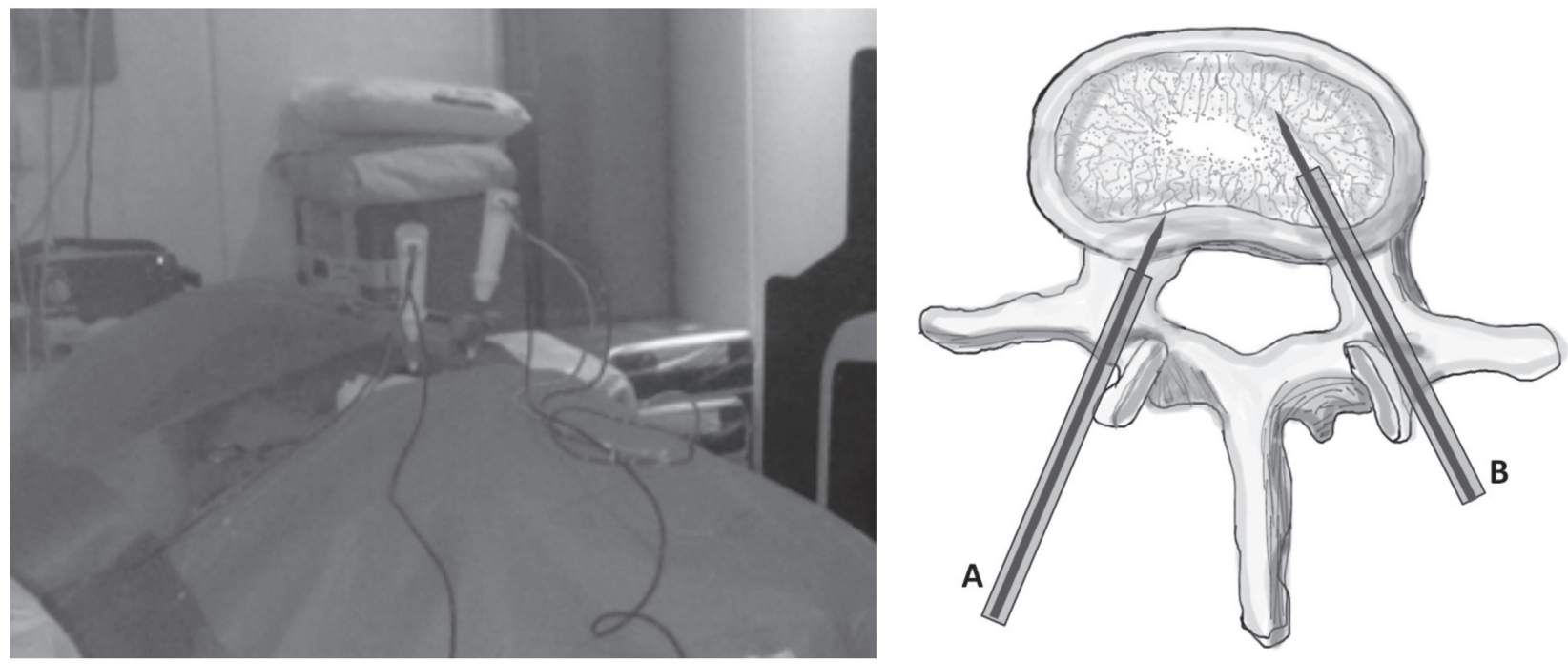

Fig. 1. Agujas de radiofrecuencia posicionadas para la ablación. Se aprecia el distinto grado de profundidad de ambas, la más superficial a la altura del muro posterior para el control de la temperatura (A) la más profunda posicionada para la ablación entre el tercio anterior y medio del cuerpo vertebral (B).

seguimiento clínico ni radiológico posterior en uno de los pacientes.

En los tres pacientes en los que se realizaron controles evolutivos, con pruebas de imagen (TC, RM), no se evidenció progresión local de las lesiones. En un paciente (metástasis pulmonar en L5) se realizó un estudio PET-TC a los 9 meses, que no mostró criterios de actividad tumoral en la vértebra tratada.

\section{DISCUSIÓN}

El tratamiento con radioterapia constituye, en combinación con las diferentes estrategias analgésicas, la pauta de manejo habitual en pacientes con dolor por metástasis óseas no complicadas. Sin embargo, aunque hasta un 70-80\% de los pacientes presentan mejoría del dolor tras la radioterapia, solo un 30\% presentan una resolución com-

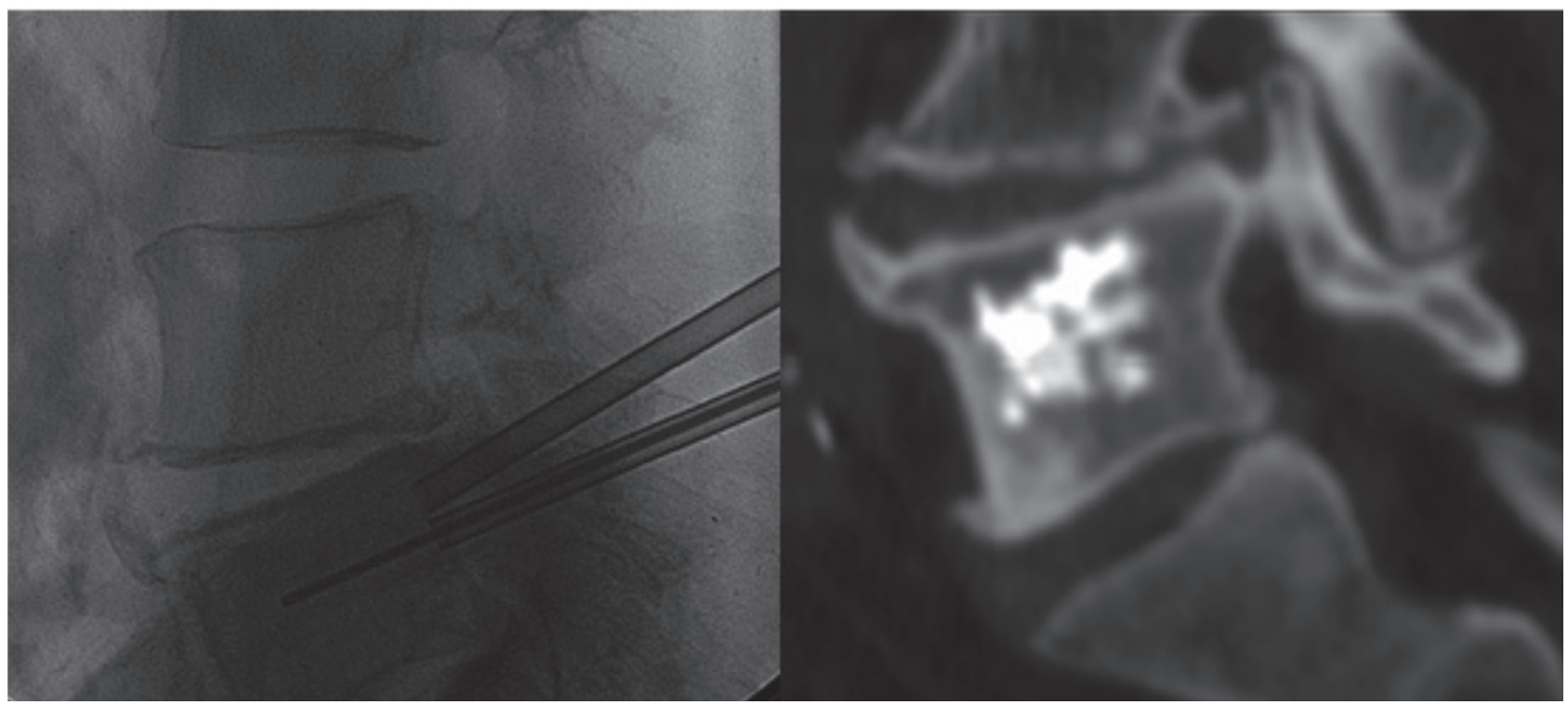

Fig. 2. Proyección lateral de columna lumbar, en la que identifica el equipo de cifoplastia a la altura de L5, en posición para la realización de la técnica, con el fiador empleado para efectuar el intercambio de las agujas de acceso óseo por el equipo de cifoplastia. Reconstrucción sagital TC de la columna lumbar tras el procedimiento, observándose el material de cifoplastia en el cuerpo vertebral de L5. 
pleta del mismo (1). El periodo medio de latencia para apreciar el efecto analgésico de la radioterapia es de dos semanas, con un máximo beneficio que suele ocurrir a las 12-20 semanas (2). Además, en ocasiones, los pacientes que tienen enfermedad recurrente en una localización previamente tratada con radioterapia no pueden ser retratados por limitaciones de tolerancia tisular.

Las técnicas percutáneas mínimamente invasivas de soporte vertebral (vertebroplastia y cifoplastia) se han utilizado en los últimos años en el tratamiento del dolor por metástasis vertebrales, con mejoría rápida del dolor y con buenos resultados en el seguimiento. Bouza y cols. (3), en un metaanálisis sobre cifoplastia con balón en fracturas malignas espinales, analizaron 7 estudios ( 3 retrospectivos y 4 prospectivos), con un total de 306 pacientes, en los que se observó una reducción rápida del dolor tras el procedimiento, con una mejoría en la capacidad funcional y la calidad de vida en la mayoría de los pacientes. El efecto analgésico de la técnica estaría en relación con la estabilización de microfracturas, y con la destrucción de terminaciones nerviosas por la citotoxicidad asociada al polimetilmetacrilato (4).

El mecanismo propuesto en la disminución del dolor en la RF está en relación con la inhibición de la transmisión tras la destrucción de las fibras nerviosas sensitivas del periostio y la cortical ósea. Adicionalmente es posible obtener una reducción del volumen de la lesión con destrucción de células tumorales, que producen citoquinas estimulantes, como el factor de necrosis tumoral alfa e interleucinas, y la inhibición de la actividad osteoclástica (5). Existen, a este respecto, varios estudios que demuestran una mejoría significativa del dolor tras el tratamiento con RF de metástasis óseas (6-8). En el estudio multicéntrico de Goetz y cols. (7) fueron tratados con RF 43 pacientes con metástasis óseas dolorosas refractarias a tratamientos habituales; $95 \%$ de estos pacientes mostraron una reducción significativa del dolor y del uso de analgésicos en las 24 semanas de seguimiento, con solo mínimas complicaciones.

Se dispone en la actualidad de los resultados del tratamiento combinado con RF y vertebroplastia o cifoplastia en series de casos, que sugieren un posible efecto sinérgico de ambas técnicas (8-13). La realización de RF, previa a la cementación, conlleva una teórica disminución del riesgo de extravasación del cemento, al destruir tejido neoplásico y generar una cavidad intralesional, minimizando además los riesgos de migración de masa tumoral al canal raquídeo en casos de lesión asociada de muro posterior, o al sector venoso por su capacidad de inducir fenómenos de trombosis en los plexos venosos intravertebrales (10). La cifoplastia o vertebroplastia aportan el beneficio adicional relacionado con los efectos analgésicos inmediatos y con la estabilización vertebral.

En nuestra experiencia preliminar, el tratamiento combinado se realizó sin complicaciones a pesar de existir lesión lítica del muro posterior en dos casos. Desde el punto de vista clínico, los resultados son buenos con mejoría del dolor en las primeras 24 horas en todos los pacientes, resultados similares a los obtenidos por otros autores (8-13). En lo relativo a los aspectos técnicos, la combinación de ambas técnicas no incrementa la complejidad del procedimiento ya que el acceso transpedicular empleado, para la realización de la cifoplastia, es el mismo que se requiere para la realización de la RF previa, con la excepción de que el acceso óseo inicial para efectuar la ablación se realizó con agujas de $11 \mathrm{G}$, que posteriormente se intercambian por el sistema de acceso de cifoplastia. Esta variación técnica está relacionada con la necesidad de establecer un margen de seguridad de al menos $1 \mathrm{~cm}$ entre la punta activa y el trocar de acceso, para evitar lesiones térmicas en el trayecto, existiendo en la actualidad un número limitado de referencias del dispositivo de RF (longitudes de aguja-puntas activas) que limitan la compatibilidad.

La posibilidad de lesión del tejido nervioso (médula y/o raíces) es elevada, con temperaturas superiores a $\operatorname{los} 45^{\circ} \mathrm{C}$ (14). Al ejercer la cortical ósea un efecto de aislante térmico, sobre las estructuras anatómicas adyacentes, existe un riesgo teórico incrementado de lesión del tejido nervioso en aquellos casos con afectación lítica de muro posterior, tal como sucedía en dos de nuestros pacientes. El estudio de Adachi y cols. (15) describe la colocación de un sensor térmico cerca del saco tecal, para monitorizar la temperatura y así evitar el daño térmico neural. El uso simultáneo de dos agujas de RF, empleando una de ellas como sensor térmico posicionado a la altura del muro posterior mientras se efectúa la ablación, posee limitaciones en lo relativo a un registro eficiente de las variaciones térmicas en el canal raquídeo. No obstante, este diseño nos permitió una monitorización simultánea de temperatura en la proximidad del muro, que no superó en ningún caso $1^{\circ} \mathrm{C}$ sobre la temperatura basal.

Con respecto a la elección de sistemas de RF con aguja, frente a dispositivos tipo paraguas, su empleo en tejido óseo permite un mejor acceso y control del punto de inserción final-ablación, minimizando los riesgos relacionados con dificultades en la retirada posterior del sistema y con la lesión térmica de estructuras neurales.

Esperamos disponer en el futuro de sistemas de acceso específico para la realización simultanea de la técnica, que en nuestra opinión debería incluir mayor número de longitudes de aguja para los distintos tamaños de punta activa, y sensores térmicos, bien a lo largo del dispositivo de ablación o de inserción independiente, que permitan un mejor control de la temperatura en la vecindad del canal durante el tratamiento.

La limitación principal de este estudio es el escaso número de pacientes y su periodo de seguimiento; no obstante nuestros resultados preliminares sugieren que el 
empleo combinado de radiofrecuencia seguida de cifoplastia, en sesión única, podría tener un efecto sinérgico capaz de mejorar el control analgésico de pacientes con mala respuesta al tratamiento médico y radioterápico, e incluso probablemente, contribuir al control tumoral local, sin que la realización conjunta de ambas técnicas conlleve una mayor complejidad.

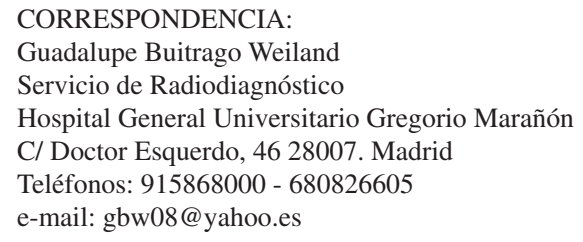

\section{BIBLIOGRAFÍA}

1. Agarawal JP, Swangsilpa T, van der Linden Y, et al. The role of external beam radiotherapy in the management of bone metastases. Clin Oncol. 2006;18:747-60.

2. Janjan NA. Radiation for bone metastases: conventional techniques and the role of systemic radiopharmaceuticals. J Cancer. 1997;80:1628-45.

3. Bouza C, López-Cuadrado T, Cediel P, et al. Balloon kyphoplasty in malignant spinal fractures: a systematic review and meta-analysis. BMC Palliat Care. 2009;8:12.

4. Ofluoglu O. Minimally invasive management of spinal metastases. Orthop Clin North Am. 2009;40:155-68.

5. Mannion RJ, Woolf CJ. Pain mechanisms and management: a central perspective. Clin J Pain. 2000;16:144-56.
6. Thanos L, Mylona S, Galani P, et al. Radiofrequency ablation of osseous metastases for the palliation of pain. Skeletal Radiol. 2008;37:189-94.

7. Goetz MP, Callstrom MR, Charboneau JW, et al. Percutaneous image-guided radiofrequency ablation of painful metastases involving bone: a multicenter study. J Clin Oncol. 2004;22:300-6.

8. Munk PL, Rashid F, Heran MK, et al. Combined cementoplasty and radiofrequency ablation in the treatment of painful neoplastic lesions of bone. J Vasc Interv Radiol. 2009;20:903-11.

9. Hoffman RT, Jakobs T, Trumm C, et al. Radiofrequency ablation in combination with osteoplasty in the treatment of painful metastatic bone diseases. J Vas Interv Radiol. 2008;19:419-25.

10. Schaefer O, Lohrmann C, Herling M, et al. Combined radiofrequency thermal ablation and percutaneous cementoplasty treatment of a pathological fracture. J Vasc Interv Radiol. 2002; $13: 1047-50$.

11. Toyota N, Naito A, Kakizawa H, et al. Radiofrequency ablation therapy combined with cementoplasty for painful bone metastases: initial experience. Cadiovasc Intervent Radiol. 2005;28:578-83.

12. Halpin RJ, Bendok BR, Sat KT, et al. Combination treatment of vertebral metastases using image guided percutaneous radiofrequency ablation and vertebroplasty: a case report. Surg Neurol. 2005;63:469-75.

13. Lane MD, Le HB, Lee $\mathrm{S}$, et al. Combination radiofrequency ablation and cementoplasty for palliative treatment of painful neoplastic bone metastasis: experience with 53 treated lesions in 36 patients. Skeletal Radiol. 2011;40:25-32.

14. Froese G, Das R, Dunscombe P. The sensivity of the thoracolumbar spinal cord of the mouse to hyperthermia. Radiat Res. 1991;125:173-80.

15. Adachi A, Kaminou T, Ogawa Tet al. Heat distribution in the spinal canal during radiofrequency ablation for vertebral lesions: study in swine. Radiology. 2008;247:374-80. 